Pure and Applied Mathematics Quarterly

Volume 10, Number 2

(Special Issue: In memory of

Andrey Todorov, Part 3 of 3 )

$325-354,2014$

\title{
Holography Principle for Twistor Spaces
}

\author{
Misha Verbitsky ${ }^{1}$
}

\begin{abstract}
Let $S$ be a smooth rational curve on a complex manifold $M$. It is called ample if its normal bundle is positive: $N S=\bigoplus \mathcal{O}\left(i_{k}\right), i_{k}>0$. We assume that $M$ is covered by smooth holomorphic deformations of $S$. The basic example of such a manifold is a twistor space of a hyperkähler or a 4dimensional anti-selfdual Riemannian manifold $X$ (not necessarily compact). We prove "a holography principle" for such a manifold: any meromorphic function defined in a neighbourhood $U$ of $S$ can be extended to $M$, and any section of a holomorphic line bundle can be extended from $U$ to $M$. This is used to define the notion of a Moishezon twistor space: this is a twistor space admitting a holomorphic embedding to a Moishezon variety $M^{\prime}$. We show that this property is local on $X$, and the variety $M^{\prime}$ is unique up to birational transform. We prove that the twistor spaces of hyperkähler manifolds obtained by hyperkähler reduction of flat quaternionic-Hermitian spaces by the action of reductive Lie groups (such as Nakajima's quiver varieties) are always Moishezon.
\end{abstract}

Keywords: Quiver varieties, hyperkähler reduction, twistor space, rational curve, quasi-line, Moishezon manifold.

Received November 25, 2012.

${ }^{1}$ Partially supported by RFBR grants 12-01-00944-, 10-01-93113-NCNIL-a, and AG Laboratory NRI-HSE, RF government grant, ag. 11.G34.31.0023. 


\section{Contents}

1 Introduction

1.1 Quasilines on complex manifolds . . . . . . . . . . . . . . . . 327

1.2 Holography principle . . . . . . . . . . . . . . . . . 328

1.3 Moishezon twistor spaces . . . . . . . . . . . . . . . . . . 329

2 Twistor spaces and ample rational curves $\quad 330$

2.1 Hyperkähler manifolds . . . . . . . . . . . . . . . . . . . . . 330

2.2 Twistor spaces . . . . . . . . . . . . . . . . . . 332

2.3 Geometry of twistor spaces . . . . . . . . . . . . . 333

2.4 Quasilines and ample curves in the twistor spaces . . . . . . . . . . . 334

$3 \quad$ Holography principle for manifolds with ample rational curves $\quad 335$

3.1 Holography principle for line bundles . . . . . . . . . . . . . . . . 335

3.2 The local holography principle . . . . . . . . . . . . . 336

3.3 Holography principle for meromorphic functions . . . . . . . . . . . . 337

4 Moishezon twistor spaces

4.1 Vector bundles in a neighbourhood of an ample curve . . . . . . . . . 338

4.2 Algebraic dimension of the field of meromorphic functions . . . . . . . . . 340

4.3 Moishezon manifolds and ample rational curves . . . . . . . . . . . . . . 341

4.4 Moishezon twistor spaces f . . . . . . . . . . . . . . . . 342

4.5 Twistor spaces and hyperkähler reduction . . . . . . . . . . . . . . . . 344

5 Appendix: Formal geometry and holography principle (by Dmitry Kaledin) 348 


\section{Introduction}

\subsection{Quasilines on complex manifolds}

The present paper was written as an attempt to answer the following question. Let $S \subset M$ be a smooth rational curve in a complex manifold, with normal bundle $N S$ isomorphic to $\mathcal{O}(1)^{n}{ }^{1}$ Is there a notion of a normal form for a tubular neighbourhood of such a curve?

When the normal bundle is $\mathcal{O}(-1)^{n}$ instead of $\mathcal{O}(1)^{n}$, a tubular neighbourhood of the curve has a normal form, obtained by blowing down this curve to a point, and taking a sufficiently small Stein neighbourhood of this point in the corresponding singular variety. When $N S=\mathcal{O}(1)^{n}$, no such normal form can be obtained. In fact, the birational type of the manifold can be reconstructed from a complex analytic (and even formal) neighbourhood of $S$. This was known already to Hartshorne ([Har, Theorem 6.7]).

However, one can associate with a quasiline an infinite-dimensional bundle $\hat{\mathcal{O}}_{S}(M)$ over $S \cong \mathbb{C} P^{1}$, with

$$
\hat{\mathcal{O}}_{S}(M)=\lim _{\leftarrow} \mathcal{O}_{M} / I_{S}^{n} \mathcal{O}_{M}
$$

where $I_{S}$ is an ideal sheaf of $S$. This bundle is called a formal completion of $M$ at $S$.

It is not hard to observe that the space of sections $H^{0}\left(S, \hat{\mathcal{O}}_{S}(M)\right)$ is finitedimensional, and, moreover, $H^{0}\left(S, \hat{\mathcal{O}}_{S}(M) \otimes_{\mathcal{O}_{S}} \mathcal{O}(i)\right)$ is finite-dimensional for each $i$ (Proposition 4.1). For a long time, we expected that the algebra $A_{S}:=$ $\bigoplus_{i} H^{0}\left(S, \hat{\mathcal{O}}_{S}(M) \otimes_{\mathcal{O}_{S}} \mathcal{O}(i)\right)$ would provide a sort of an algebraic "normal form" of $S$ in $M$, in such a way that the algebraic structure on $\hat{\mathcal{O}}_{S}(M)$ can be reconstructed from this ring.

In this paper, we show that this approach works when $M$ is a Moishezon manifold, and $M$ can be reconstructed from the ring $A_{S}$, up to birational isomorphism (Subsection 4.3; this is not very surprising due to the above-mentioned theorem of Hartshorne, [Har, Theorem 6.7]).

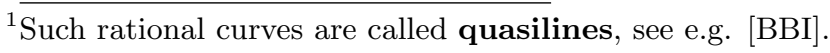


For non-Moishezon $M$, such as a twistor space of a simply connected, compact hyperkähler manifold, this conjecture is spectacularly wrong. In this case, $A_{S}=\bigoplus_{i} H^{0}\left(\mathbb{C} P^{1}, \mathcal{O}(i)\right)$, and this ring has no information about $M$ whatsoever (Proposition 2.13).

\subsection{Holography principle}

The main technical tool of the present paper is the following theorem, called the holography principle. Recall that an ample rational curve on a complex variety $M$ is a smooth curve $S \cong \mathbb{C} P^{1} \subset M$ such that the normal bundle $N S$ is decomposed as $N S=\bigoplus_{k} \mathcal{O}\left(i_{k}\right)$, with all $i_{k}>0$.

Theorem 1.1: Let $S \subset M$ be an ample curve in a simply connected complex manifold, and $U$ its connected neighbourhood. Suppose that $M$ is covered by smooth complex-analytic deformations of $S$. Then

(i) For any holomorphic vector bundle $B$ on $M$, the restriction map

$$
H^{0}(M, B) \longrightarrow H^{0}(U, B)
$$

is an isomorphism.

(ii) Let $\operatorname{Mer}(M), \operatorname{Mer}(U)$ be the fields of meromorphic functions on $M$ and $U$. Then the restriction map $\operatorname{Mer}(M) \longrightarrow \operatorname{Mer}(U)$ is an isomorphism.

Proof: For line bundles, Theorem 1.1 (i) is implied by Theorem 3.1. For general vector bundles, an elegant argument is given by D. Kaledin in the appendix to this paper (Section 5). Holography principle for meromorphic functions is proven in Theorem 3.4.

The holography principle is not very surprising of one looks at the neighbourhood of $S$ from the point of view of complex analysis. The normal bundle to $S$ is obviously positive. Choose a Hermitian metric $h$ on a neighbourhood of $S$ such that the Chern connection on $N S$ induced by $h$ has positive curvature. Let 
$d_{S}: M \longrightarrow \mathbb{R}$ be the Riemannian distance to $S$ in this Hermitian metric. Since $d_{S}$ around $S$ is close to the distance in $N S$, the form $d d^{c} d_{S}$ has $n-1$ positive and 1 negative eigenvalue in a sufficiently small neighbourhood of $S([\mathrm{D}])$. This means that $S$ has a neighbourhood $U$ with a smooth boundary $\partial U$ such that the Levi form on $\partial U$ has one negative and $\operatorname{dim} M-2$ positive eigenvalues. Then, a holomorphic function defined on an open subset of $U$ and continuous on $\partial U$ can be extended outside of a boundary; at least, this is the expectation one has from the solution of the Levi problem.

\subsection{Moishezon twistor spaces}

Definition 1.2: Let $M$ be a compact complex manifold. Define the algebraic dimension as $a(M):=\operatorname{deg}_{t r} \operatorname{Mer}(M)$, where $\operatorname{deg}_{t r} \operatorname{Mer}(M)$ denotes the transcendence degree of the field of global meromorphic functions on $M$.

Definition 1.3: A Moishezon variety is a compact complex variety satisfying $a(M)=\operatorname{dim} M$.

The notion of a Moishezon manifold, as it is usually stated, makes no sense for non-compact varieties. Indeed, $\operatorname{deg}_{t r} \operatorname{Mer}(M)=\infty$ even if $M$ is an open disk.

However, when $M$ contains an ample curve, the situation changes drastically.

Theorem 1.4: Let $M$ be a complex manifold containing an ample rational curve. Then $a(M) \leqslant \operatorname{dim} M$. Moreover, if $a(M)=\operatorname{dim} M$, there exists an open embedding of $M$ to a Moishezon variety $M^{\prime}$ which satisfies $\operatorname{Mer}\left(M^{\prime}\right)=\operatorname{Mer}(M)$.

Proof: See Theorem 4.8.

Let now $M$ be a twistor space of $X$. Here, $X$ can be either a hypercomplex (hyperkähler) manifold, a quaternionic, or quaternionic-Kähler manifold, or 
Riemannian anti-selfdual manifold. We are not very specific, because the only thing about $M$ which is used is existence of a large number of quasilines. The twistor spaces are complex manifold covered by quasilines, usually non-Kähler and non-quasiprojective (see Proposition 2.13 and Theorem 4.31).

Since $M=\operatorname{Tw}(X)$ is covered by quasilines which are by definition ample, we can apply Theorem 4.6, and obtain that $\operatorname{deg}_{t r} \operatorname{Mer}(M) \leqslant \operatorname{dim} M$. We call $M$ a Moishezon twistor space if $\operatorname{deg}_{t r} \operatorname{Mer}(M)=\operatorname{dim} M$. This is equivalent to an existence of an open embedding $M \longrightarrow M^{\prime}$ of $M$ to a Moishezon manifold (Theorem 1.4).

Moishezon twistor spaces for compact 4-dimensional anti-selfdual manifolds were discovered by Y.-S. Poon in $[\mathrm{P}]$, and much studied since then. Structure theorems about such manifolds were obtained by F. Campana, $[\mathrm{C}]$, and partial classification results by N. Honda (see e.g. [Ho]).

The definition given above extends the class of "Moishezon twistor manifolds" significantly. Let, for instance, $M:=V / / / G$ be a hyperkähler manifold which can be obtained using the hyperkähler reduction, where $V$ is a flat hyperkähler manifold, and $G$ a compact Lie group (such as the Nakajima quiver variety). Then the twistor space $\operatorname{Tw}(M)$ is always Moishezon (Theorem 4.29).

Acknowledgements: Many thanks to Hans-Joachim Hein, Claude LeBrun, Nobuhiro Honda and Dima Kaledin for interesting discussions on the subject of this article.

\section{Twistor spaces and ample rational curves}

\subsection{Hyperkähler manifolds}

Definition 2.1: Let $M$ be a manifold, and $I, J, K \in \operatorname{End}(T M)$ endomorphisms of the tangent bundle satisfying the quaternionic relation

$$
I^{2}=J^{2}=K^{2}=I J K=-\operatorname{Id}_{T M} .
$$

The manifold $(M, I, J, K)$ is called hypercomplex if the almost complex structures $I, J, K$ are integrable. If, in addition, $M$ is equipped with a Riemannian 
metric $g$ which is Kähler with respect to $I, J, K$, the manifold $(M, I, J, K, g)$ is called hyperkähler.

Consider the Kähler forms $\omega_{I}, \omega_{J}, \omega_{K}$ on $M$ :

$$
\omega_{I}(\cdot, \cdot):=g(\cdot, I \cdot), \quad \omega_{J}(\cdot, \cdot):=g(\cdot, J \cdot), \quad \omega_{K}(\cdot, \cdot):=g(\cdot, K \cdot) .
$$

An elementary linear-algebraic calculation implies that the 2 -form $\Omega:=\omega_{J}+$ $\sqrt{-1} \omega_{K}$ is of Hodge type $(2,0)$ on $(M, I)$. This form is clearly closed and nondegenerate, hence it is a holomorphic symplectic form.

In algebraic geometry, the word "hyperkähler" is essentially synonymous with "holomorphically symplectic", due to the following theorem, which is implied by Yau's solution of Calabi conjecture.

Theorem 2.2: Let $(M, I)$ be a compact, Kähler, holomorphically symplectic manifold. Then there exists a unique hyperkähler metric on $(M, I)$ with the same Kähler class.

Proof: See $[Y]$, [Bes].

Remark 2.3: The hyperkähler metric is unique, but there could be several hyperkähler structures compatible with a given hyperkähler metric on $(M, I)$, if the holonomy of its Levi-Civita connection is strictly less than $\operatorname{Sp}(n)$.

Definition 2.4: Let $M$ be a hypercomplex manifold, and $L$ a quaternion satisfying $L^{2}=-1$. Then $L=a I+b J+c K, a^{2}+b^{2}+c^{2}=1$. The corresponding complex structure on $M$ is called an induced complex structure. The space $M$, considered as a complex manifold, is denoted by $(M, L)$. The set of induced complex structures is naturally identified with $S^{2}$, which we often consider as $\mathbb{C} P^{1}$ with the standard complex structure.

Definition 2.5: ([V1]) Let $X \subset M$ be a closed subset of a hyperkähler manifold $M$. Then $X$ is called trianalytic if $X$ is a complex analytic subset of $(M, L)$ for 
every induced complex structure $L$.

Trianalytic subvarieties were a subject of a long study. Most importantly, consider a generic induced complex structure $L$ on $M$. Then all closed complex subvarieties of $(M, L)$ are trianalytic. Moreover, a trianalytic subvariety can be canonically desingularized ([V2]), and this desingularization is hyperkähler.

Theorem 2.6: ([V1], [V3]) Let $(M, I, J, K)$ be a hyperkähler manifold (not necessarily compact). Then there exists a countable subset $R \subset \mathbb{C} P^{1}$, such that for any induced complex structure $L \notin R$, all compact complex subvarieties of $(M, L)$ are trianalytic.

Remark 2.7: For hypercomplex manifolds, Theorem 2.6 is (generally speaking) false, though for manifolds with trivial canonical bundle a weaker form of this result was obtained ([SV]).

\section{$2.2 \quad$ Twistor spaces}

Definition 2.8: Let $M$ be a Riemannian 4-manifold. Consider the action of the Hodge $*$-operator: $*: \Lambda^{2} M \longrightarrow \Lambda^{2} M$. Since $*^{2}=1$, the eigenvalues are \pm 1 , and one has a decomposition $\Lambda^{2} M=\Lambda^{+} M \oplus \Lambda^{-} M$ onto selfdual $(* \eta=\eta)$ and anti-selfdual $(* \eta=-\eta)$ forms.

Remark 2.9: If one changes the orientation of $M$, leaving metric the same, $\Lambda^{+} M$ and $\Lambda^{-} M$ are exchanged. Therefore, their dimensions are equal, and $\operatorname{dim} \Lambda^{2} M=$ 6 implies $\operatorname{dim} \Lambda^{ \pm}(M)=3$.

Remark 2.10: Using the isomorphism $\Lambda^{2} M=\mathfrak{s o}(T M)$, we interpret $\eta \in \Lambda_{m}^{2} M$ as an endomorphisms of $T_{m} M$. Then the unit vectors $\eta \in \Lambda_{m}^{+} M$ correspond to oriented, orthogonal complex structures on $T_{m} M$. 
Definition 2.11: Let $\operatorname{Tw}(M):=S \Lambda^{+} M$ be the set of unit vectors in $\Lambda^{+} M$. At each point $(m, s) \in \operatorname{Tw}(M)$, consider the decomposition $T_{m, s} \operatorname{Tw}(M)=T_{m} M \oplus$ $T_{s} S \Lambda_{m}^{+} M$, induced by the Levi-Civita connection. Let $I_{s}$ be the complex structure on $T_{m} M$ induced by $s, I_{S \Lambda_{m}^{+} M}$ the complex structure on $S \Lambda_{m}^{+} M=S^{2}$ induced by the metric and orientation, and

$$
\mathcal{I}: T_{m, s} \operatorname{Tw}(M) \longrightarrow T_{m, s} \operatorname{Tw}(M)
$$

be equal to $\mathcal{I}_{s} \oplus I_{S \Lambda_{m}^{+} M}$. An almost complex manifold $(\operatorname{Tw}(M), \mathcal{I})$ is called the twistor space of $M$.

Given a hyperkähler or hypercomplex manifold (Definition 2.1), one defines its twistor space in a similar manner.

Definition 2.12: A twistor space $\operatorname{Tw}(M)$ of a hypercomplex manifold $M$ is $S^{2} \times M$ equipped with a complex structure which is defined as follows. Consider the complex structure $I_{m}: T_{m} M \rightarrow T_{m} M$ on $M$ induced by $J \in S^{2} \subset \mathbb{H}$. Let $I_{J}$ denote the complex structure on $S^{2}=\mathbb{C} P^{1}$. The operator $I_{\mathrm{Tw}}=I_{m} \oplus I_{J}$ : $T_{x} \operatorname{Tw}(M) \rightarrow T_{x} \operatorname{Tw}(M)$ satisfies $I_{\mathrm{Tw}}^{2}=-\mathrm{Id}$. It defines an almost complex structure on $\operatorname{Tw}(M)$.

The almost complex structure on the twistor space of a Riemannian 4-manifold $X$ is integrable whenever $X$ is anti-selfdual ([AHS]) For a hypercomplex manifold it is integrable as well $([\mathrm{K}])$. Twistor spaces are the main example of the geometries we are working with.

\section{$2.3 \quad$ Geometry of twistor spaces}

Proposition 2.13: Let $\operatorname{Tw}(M)$ be a twistor space of a compact hyperkähler manifold. Then

(i) $\operatorname{Tw}(M)$ is non-Kähler. 
(ii) The algebraic dimension of $\mathrm{Tw}(M)$ is 1 .

Proof of (i): Let $\omega$ be the standard Hermitian form of $\operatorname{Tw}(M)$. Then $d d^{c} \omega$ is a positive $(2,2)$-form $([\mathrm{KV},(8.2)])$. For any Kähler form $\omega_{0}$ on $\operatorname{Tw}(M)$, this would imply

$$
\int_{\operatorname{Tw}(M)} d\left(\omega_{0}^{\operatorname{dim}_{\mathbb{C}} M-1} \wedge d^{c} \omega\right)=\int_{\mathrm{Tw}(M)} \omega_{0}^{\operatorname{dim}_{\mathbb{C}} M-1} \wedge d d^{c} \omega>0
$$

which is impossible by Stokes' theorem.

Proof of (ii): See Theorem 4.11.

\subsection{Quasilines and ample curves in the twistor spaces}

Definition 2.14: An ample rational curve on a complex manifold $M$ is a smooth curve $S \cong \mathbb{C} P^{1} \subset M$ such that its normal bundle $N S$ satisfies $N S=$ $\bigoplus_{k=1}^{n-1} \mathcal{O}\left(i_{k}\right)$, with all $i_{k}>0$ (see [Ko]). It is called a quasiline if all $i_{k}=1$.

Claim 2.15: Let $M$ be a twistor space of a hyperkähler or 4-dimensional ASD manifold, $m \in M$ a point, and $S_{m}$ the corresponding $S^{2}$ in $\operatorname{Tw}(M)=\mathbb{C} P^{1} \times M$. Then $S_{m}$ is a quasiline.

Proof: Since the claim is essentially infinitesimal, it suffices to check it when $M$ is flat. Then $\operatorname{Tw}(M)=\operatorname{Tot}\left(\mathcal{O}(1)^{\oplus 2 n}\right) \cong \mathbb{C} P^{2 n+1} \backslash \mathbb{C} P^{2 n-1}$, and $S_{m}$ is a section of $\mathcal{O}(1)^{\oplus 2 n}$.

Existence of quasilines in twistor spaces is a very strong condition, and can be used to obtain all kinds of geometric information; for example, see [C] and [V4]. 


\section{Holography principle for manifolds with ample ra- tional curves}

\subsection{Holography principle for line bundles}

Throughout this paper, all neighbourhoods and manifolds are silently assumed to be connected. One of the main results of the present paper is the following theorem.

Theorem 3.1: (holography principle for line bundles) Let $S \subset M$ be an ample rational curve in a simply connected complex manifold, which is covered by smooth, ample deformations of $S$, and $L$ a holomorphic line bundle on $M$. Consider an open neighbourhood $U \supset S$. Then the restriction map $H^{0}(M, L) \longrightarrow H^{0}(U, L)$ is an isomorphism.

We deduce Theorem 3.1 from the following local result (Proposition 3.3).

Remark 3.2: Since $S$ is an ample curve, $S$ can be deformed in any normal direction. Therefore, there exists an open neighbourhood $U \supset S$ which is contained in a union of the set $\mathfrak{S}$ of all smooth, ample deformations of $S$ intersecting $S$. Further on, we choose this neighbourhood in such a manner that any $S_{1} \in \mathfrak{S}$ can be connected to $S$ by a continuous family of deformations intersecting $S$.

Proposition 3.3: Let $S \subset M$ be an ample rational curve in a connected complex manifold, which is covered by deformations of $S$, and $L$ a holomorphic line bundle on $M$. Consider a neighbourhood $U \supset S$ which is is contained in a union of the set of all deformations of $S$ intersecting $S$ (Remark 3.2). Then for any smaller open neighbourhood $V \subset U$ of $S$, the restriction map $H^{0}(U, L) \longrightarrow H^{0}(V, L)$ is an isomorphism. 


\section{Proof of an implication "Proposition $3.3 \Rightarrow$ Theorem 3.1".}

Step 1: Choose a continuous, connected family $S_{b}$ of ample curves parametrized by $B$ such that $\bigcup_{b \in B} S_{b}=M$, and choose a tubular neighbourhood $U_{b}$ for each $S_{b}$, continuously depending on $b$. Then the intersection $U_{b} \cap U_{b^{\prime}}$ for sufficiently close $b, b^{\prime}$ always contains $S_{b}$ and $S_{b^{\prime}}$. By Proposition 3.3, $U_{b}$ can be chosen in such a way that $H^{0}\left(U_{b} \cap U_{b^{\prime}}, L\right)=H^{0}\left(U_{b}, L\right)=H^{0}\left(U_{b^{\prime}}, L\right)$.

Step 2: Since $B$ is connected, all the spaces $H^{0}\left(U_{b}, L\right)$ are isomorphic, and these isomorphisms are compatible with the restrictions to the intersections $U_{b} \cap$ $U_{b^{\prime}}$. Let now $f \in H^{0}\left(U_{b}, L\right)$, and let $\tilde{M}_{f}$ be the domain of holomorphy for $f$, that is, a maximal domain (non-ramified over $M$ ) such that $f$ admits a holomorphic extension to $\tilde{M}_{f}$. Since $\cup U_{b}=M$, and $f$ can be holomorphically extended to any $U_{b}$, the domain $\tilde{M}_{f}$ is a covering of $M$. Now, Theorem 3.1 follows, because $M$ is simply connected.

\subsection{The local holography principle}

To prove Theorem 3.1 it remains to prove Proposition 3.3. If $\left.\operatorname{deg} L\right|_{S}<0$, the statement of Proposition 3.3 is vacuous. Therefore, we may always assume that $\left.\operatorname{deg} L\right|_{S} \geqslant 0$, hence $\left.l\right|_{S}$ is generated by global sections. Let $S(M)$ be the space of deformations of the ample curve $S$ which remain smooth, and $S S(M)$ the space of pairs $\left\{\left(x, S_{1}\right): S_{1} \in S(M), x \in S_{1}\right\}$. Consider the natural forgetful maps $\tau_{1}: S S(M) \longrightarrow M, \tau_{2}: S S(M) \longrightarrow S(M)$, and let $E$ be the bundle $\tau_{2 *} \tau_{1}^{*} L$ on $S(M)$. Denote by $\operatorname{deg}_{S} L$ the degree of the restriction of $L$ to $S$. Since $\operatorname{dim} H^{0}\left(S_{1}, L\right)=\operatorname{deg} L+1, E$ is a $(\operatorname{deg} L+1)$-dimensional vector bundle. Given a section $f$ of $L$ on $M$, denote the corresponding section of $E$ by $\tilde{f}:=\tau_{2 *} \tau_{1}^{*} f$. When $\operatorname{deg}_{S} L=d$, the value of $\tilde{f}$ at $S_{1}$ is uniquely determined by the restriction of $f$ to any $d+1$ distinct points of $S_{1}$. Indeed, $\left.E\right|_{S_{1}}$ is $d+1$-dimensional, and any section $h \in H^{0}\left(S_{1}, L\right)$ is uniquely determined by its values in $d+1$ points. This gives a map

$$
\left.L\right|_{z_{1}} \times\left. L\right|_{z_{2}} \times \ldots \times\left. L\right|_{z_{d+1}} \stackrel{\sim}{\longrightarrow} H^{0}\left(S_{1}, L\right) .
$$

Now, let $f$ be a section of $L$ on $V$. For any $S_{1} \in S(M)$ intersecting $V$, we choose $d_{1}$ distinct points $z_{1}, \ldots, z_{d+1} \in S_{1} \cap V$, and consider the section 
$\varphi\left(\left.f\right|_{z_{1}},\left.f\right|_{z_{2}}, \ldots,\left.f\right|_{z_{d+1}}\right) \in H^{0}\left(S_{1}, L\right)$ defined using (3.1). When $S_{1} \subset V$, this section is independent from the choice of $z_{1}, \ldots, z_{d+1} \in S_{1}$. Let $R_{V}$ be a connected component of the set of all $S_{1} \in S(M)$ intersecting $V$ and containing $S$. Since the map

$$
\left(z_{1}, \ldots, z_{d+1}\right) \longrightarrow \varphi\left(\left.f\right|_{z_{1}},\left.f\right|_{z_{2}}, \ldots,\left.f\right|_{z_{d+1}}\right)
$$

is holomorphic and independent from the choice of $z_{1}, \ldots, z_{d+1}$ on an open subset of $R_{V}$, it is independent of $z_{1}, \ldots, z_{d+1}$ everywhere on $R_{V}$. This gives a section $\tilde{f} \in H^{0}\left(R_{V}, E\right)$ extending the section $\tilde{f}:=\tau_{2 *} \tau_{1}^{*} f \in H^{0}(S(V), E)$.

By construction, $U$ is contained in a connected part $U_{1}$ of the union of all deformations of $S$ intersecting $V$. To extend $f$ from $V$ to $U$, we use $\tilde{f}$ to obtain an extension of $f$ to $U_{1}$, as follows.

Any section $g \in H^{0}\left(R_{V}, E\right)$ gives a function $\psi_{g}$ mapping a pair $\left(x, S_{1}\right)$, $x \in S_{1} \in R_{V}$ to $\left.\left.g\left(S_{1}\right)\right|_{x} \in L\right|_{x}$. For the section $\tilde{f}$ constructed above, $\psi_{\tilde{f}}\left(x, S_{1}\right)$ is independent from the choice of $S_{1}$ whenever $S_{1}$ lies in $V$. The same analytic continuation argument as above implies that $\psi_{\tilde{f}}\left(x, S_{1}\right)$ is independent of $S_{1}$ everywhere. For any $x \in U_{1}$, the set $\left.f \in L\right|_{x}$ equal to $\psi_{\tilde{f}}\left(x, S_{1}\right)$, where $S_{1} \in R_{V}$ is an arbitrary curve passing through $x$. This gives an extension of $f$ to $U_{1}$. Proposition 3.3 is proven. We finished the proof of Theorem 3.1.

\subsection{Holography principle for meromorphic functions}

The following theorem is proven in the same way as Theorem 3.1. Given a complex variety $M$, we denote the field of meromorphic functions on $M$ by $\operatorname{Mer}(M)$.

Theorem 3.4: Let $S \subset M$ be an ample curve in a simply connected complex manifold, and $U \supset S$ a connected neighbourhood of $S$. Suppose that $M$ is covered by the union of all smooth, ample deformations of $S$. Then the restriction map $\operatorname{Mer}(M) \longrightarrow \operatorname{Mer}(U)$ is an isomorphism.

Proof: The same argument as used to deduce Theorem 3.1 from Proposition 3.3 can be used to reduce Theorem 3.4 to the following statement. 
Proposition 3.5: Let $S \subset M$ be an ample rational curve in a connected complex manifold, which is covered by smooth, ample deformations of $S$. Consider a neighbourhood $U \supset S$ which is contained in a union $\mathfrak{S}$ of all deformations of $S$ intersecting $S$ (Remark 3.2). Then for any smaller open neighbourhood $V \subset U$ of $S$, the restriction map $\operatorname{Mer}(U) \longrightarrow \operatorname{Mer}(V)$ is an isomorphism.

Proof: Define the degree $\operatorname{deg}_{S}(f)$ of a meromorphic function $f \in \operatorname{Mer}(V)$ as the degree of the pole divisor of $\left.f\right|_{S_{1}}$ for any deformation $S_{1}$ of $S$ transversal to the pole divisor of $f$. Denote by $\operatorname{Mer}_{d}(V)$ the space meromorphic functions of degree $\leqslant d$. To prove Proposition 3.5 it would suffice to show that the restriction map $\operatorname{Mer}_{d}(U) \longrightarrow \operatorname{Mer}_{d}(V)$ is an isomorphism, for all $d$.

For each rational curve $S_{1}$, a degree $\leqslant d$ meromorphic function is uniquely determined by its values in any $d+1$ distinct points on $S_{1}$. Given a meromorphic function $f \in \operatorname{Mer}_{d}(V)$, and a deformation $S_{1}$ of $S$ intersecting $V$, we can extend $\left.f\right|_{S_{1} \cap V}$ to a degree $d$ meromorphic function $f_{1}$ on $S_{1}$ by computing its values at $d+1$ distinct points $z_{1}, \ldots, z_{d+1}$ of $S_{1} \cap V$. Whenever $S_{1}$ is in $V$, this procedure gives $\left.f\right|_{S_{1}}$. By analytic continuation, the values of $f_{1}(z)$ at any $z \in S_{1}$ are independent from the choice of $z_{i}$ and $S_{1}$.

We have shown that $f_{1}$ is a well-defined meromorphic function on the union $U_{1}$ of all deformations of $S$ intersecting $V$. By construction, on $V$ we have $f_{1}=f$. Since $U_{1}$ contains $U$, this implies that $f$ can be extended from $V$ to $U$.

\section{Moishezon twistor spaces}

\subsection{Vector bundles in a neighbourhood of an ample curve}

Proposition 4.1: Let $S \subset M$ be an ample rational curve, $U \supset S$ its neighbourhood, and $B$ a holomorphic bundle on $U$. Then $H^{0}(U, B)$ is finite-dimensional.

Proof. Step 1: Let $I_{S} \subset \mathcal{O}_{U}$ be the ideal sheaf of $S$, and $J_{S}^{r}(B):=B / I_{S}^{r+1} B$ 
the sheaf of $r$-jets of the sections of $B$. Since $I_{S}^{r} / I_{S}^{r+1}=\operatorname{Sym}^{r}\left(N^{*} S\right)$, the bundle $I_{S}^{r} / I_{S}^{r+1}$ is a direct sum of $\mathcal{O}\left(k_{i}\right)$ with $k_{i}<-r$. Therefore, $H^{0}\left(I_{S}^{r} B / I_{S}^{r+1} B\right)=0$ for $r$ sufficiently big.

Step 2: The sheaf $I_{S}^{r} B$ admits a filtration $I_{S}^{r} B \supset I_{S}^{r+1} B \supset I_{S}^{r+2} B \supset \ldots$ with associated graded sheaves $I_{S}^{r} B / I_{S}^{r+1} B$ having no sections for $r \gg 0$. Therefore, $H^{0}\left(I_{S}^{r} B\right)=0$ for sufficiently byg $r$.

Step 3: If $H^{0}(U, B)$ is infinite-dimensional, the map

$$
H^{0}(U, B) \longrightarrow H^{0}\left(B / I_{S}^{r}(B)\right)
$$

cannot be injective. Then, for each $r$, there exists a non-zero section with vanishing $r$-jet: $f_{r} \in H^{0}\left(U, I_{S}^{r+1} B\right)$. This is impossible, as shown in Step 2 .

This proof is effective, and gives the following bound on the dimension of the space of sections of $B$.

Corollary 4.2: Let $S \subset M$ be an ample rational curve, $U \supset S$ its neighbourhood, and $B$ a holomorphic bundle on $U$. Then

$$
\operatorname{dim} H^{0}(U, B) \leqslant \operatorname{dim} H^{0}\left(S,\left.\bigoplus_{d} \operatorname{Sym}^{d}\left(N^{*} S\right) \otimes_{\mathcal{O}_{S}} B\right|_{S}\right) .
$$

The same argument can be applied to degree $d$ meromorphic functions. Recall that the degree $\operatorname{deg}_{S}(f)$ of a meromorphic function $f \in \operatorname{Mer}(V)$ is the degree of the pole divisor of $\left.f\right|_{S_{1}}$ for any deformation of $S$ transversal to the pole divisor of $f$. We denote the space of meromorphic functions of degree $\leqslant d$ on $U$ by $\operatorname{Mer}_{d}(U)$. 
Corollary 4.3: Let $S \subset M$ be an ample rational curve, and $U \supset S$ its neighbourhood. Then

$$
\operatorname{dim} \operatorname{Mer}_{d}(U) \leqslant \operatorname{dim} H^{0}\left(S, \operatorname{Sym}^{\leqslant d}\left(N^{*} S\right) \otimes_{\mathcal{O}_{S}} \mathcal{O}(d)\right) .
$$

\subsection{Algebraic dimension of the field of meromorphic functions}

The conormal bundle $N^{*} S$ is negative; clearly, the dimension (4.1) is maximal when $S$ is a quasiline, and $N^{*} S=\oplus \mathcal{O}(1)$. In this case, the bound (4.1) is realized for a rational line in $\mathbb{C} P^{n}$. Indeed, for a rational line $S$ in $\mathbb{C} P^{n}$, the sheaf of algebraic functions in a neighbourhood of $S$ is isomorphic to of $\bigoplus_{i} \operatorname{Sym}^{i}\left(N^{*} S\right)$. This implies the following simple numerical result.

Claim 4.4: Let $S \subset M$ be an ample rational curve, and $U \supset S$ its neighbourhood. Then

$$
\operatorname{dim} \operatorname{Mer}_{d}(U) \leqslant \operatorname{dim} H^{0}\left(\mathbb{C} P^{n}, \mathcal{O}(d)\right) .
$$

where $n=\operatorname{dim} M$.

Corollary 4.5: Let $M$ be a complex variety containing an ample rational curve. Then the transcendence degree of $\operatorname{Mer}(M)$ satisfies $\operatorname{deg}_{t r} \operatorname{Mer}(M) \leqslant \operatorname{dim} M$.

Proof: Consider the graded ring $\bigoplus_{d} \operatorname{Mer}_{d}(M)$. Since

$$
\operatorname{dim} \operatorname{Mer}_{d}(U) \leqslant \operatorname{dim} H^{0}\left(\mathbb{C} P^{n}, \mathcal{O}(d)\right),
$$

the Krull dimension of this ring is $\leqslant n$. Therefore, the transcendence degree of its ring of fractions is also bounded by $n$.

This observation is not new: it was known already to Hartshorne (in the context of formal neighbourhoods). Applied to complex analytic spaces, Hartshorne's theorem can be stated as follows. 
Theorem 4.6: Let $S \subset M$ be a connected, positive-dimensional, smooth subvariety in a complex manifold. Assume that the normal bundle of $S$ is ample. Then the transcendence degree of the field $\operatorname{Mer}(M)$ of meromorphic functions is no bigger than the dimension of $M$ :

$$
\operatorname{deg}_{t r} \operatorname{Mer}(M) \leqslant \operatorname{dim}(M)
$$

Moreover, if equality is reached, $\operatorname{Mer}(M)$ is a finitely generated extension of $\mathbb{C}$.

Proof: [Har, Theorem 6.7]; see also [KST].

\subsection{Moishezon manifolds and ample rational curves}

Hartshorne's methods are already sufficient to prove the following general result.

Proposition 4.7: Let $S \subset M$ be an ample rational curve in a simply connected complex manifold. Assume that $\operatorname{deg}_{t r} \operatorname{Mer}(M)=\operatorname{dim}(M)$. Then there exists a meromorphic map $\varphi: M \longrightarrow Z_{0}$ to an open subset of a projective variety $M$, which is bijective onto its image outside of a complex analytic subset of positive codimension.

Proof: By Hartshorne's theorem (Theorem 4.6), $\operatorname{Mer}(M)$ is a finitely generated extension of $\mathbb{C}$. Let $\xi_{1}, \ldots, \xi_{N}$ be generators of $\operatorname{Mer}(M), D_{i}$ their pole divisors, and $L_{i}:=\mathcal{O}\left(D_{i}\right)$ the corresponding line bundles. Then $\xi_{i}$ can be considered as sections of $L_{i}$, and $\xi_{1}, \ldots, \xi_{N}$ - as sections of $L:=\bigotimes_{i} L_{i}$. Consider now the subring of $\bigoplus_{d} H^{0}\left(M, L^{d}\right)$ generated by $\xi_{i}$, and let $Z$ be its spectre. Clearly, $\operatorname{dim} M=\operatorname{dim} Z, Z$ is projective, and the natural rational map $M \stackrel{\varphi}{\longrightarrow} Z$ induces an isomorphism $\operatorname{Mer}(Z) \stackrel{\sim}{\longrightarrow} \operatorname{Mer}(M)$.

Let $\tilde{M}$ be a resolution of the base set of $\varphi$, such that $\tilde{M} \stackrel{\tilde{\varphi}}{\longrightarrow} Z$ is holomorphic. If $\tilde{\varphi}$ is ramified at some divisor $D$ in $\varphi(\tilde{M})$, this divisor can be extended to $Z$ using Theorem 3.4 applied to $U=\varphi(\tilde{M}) \subset Z$. Taking the corresponding 
ramified covering $\tilde{Z}$ of $Z$, we obtain a holomorphic map from $\tilde{M}$ to $\tilde{Z}$, which is impossible, because $\operatorname{Mer}(\tilde{Z})$ is strictly bigger than $\operatorname{Mer}(Z)$, and $\operatorname{Mer}(\tilde{M})=$ $\operatorname{Mer}(Z)$. Therefore, $\tilde{\varphi}$ is bijective to its impage at its general point. Openness of its image is a general property of bimeromorphic maps.

Theorem 4.8: Let $S \subset M$ be an ample rational curve in a simply connected complex manifold. Assume that $\operatorname{deg}_{t r} \operatorname{Mer}(M)=\operatorname{dim}(M)$. Then there exists an open embedding of $M$ to a Moishezon variety.

Proof: From Proposition 4.7 we obtain a line bundle $L$ on $M$ inducing a bimeromorphic map $\varphi: M \longrightarrow Z_{0}$ to an open subset of a projective variety $Z$. Resolving the base points of the inverse map if necessary, we may assume that the inverse map $\psi: Z_{0} \longrightarrow M$ is holomorphic. Then, $M$ is obtained from $Z_{0}$ by blowing down a certain number of exceptional subvarieties $E_{i}$, obtained as common zero sets of a certain number of meromorphic functions. Applying Theorem 3.4 to $Z_{0} \subset Z$, we extend the meromorphic functions and the corresponding subvarieties $E_{i}$ and obtain closed exceptional subvarieties $E_{i}^{\prime} \subset Z$. Blowing these down, we obtain a Moishezon variety which contains $M$ as an open subset.

\subsection{Moishezon twistor spaces}

Definition 4.9: Let $M=\operatorname{Tw}(X)$ be a twistor space of a simply connected hyperkähler, hypercomplex, quaternionic or or 4-dimensional anti-selfdual manifold, not necessarily compact. We say that $M$ is a Moishezon twistor space if $\operatorname{deg}_{t r} \operatorname{Mer}(M)=\operatorname{dim}(M)$ (see Corollary 4.5).

From Theorem 4.8, we immediately obtain the following corollary.

Corollary 4.10: Let $M$ be a Moishezon twistor space. Then $M$ admits an open embedding to a Moishezon variety $M_{1}$. Moreover, $M_{1}$ is unique up to a bimeromorphic equivalence. 
Proof: The open embedding to a Moishezon variety follows from Theorem 4.8 , and its uniqueness is implied by an isomorphism $\operatorname{Mer}(M)=\operatorname{Mer}\left(M_{1}\right)$ (Theorem 3.4).

It is easy to construct an example of a twistor manifold which does not belong to this class. The twistor space of a K3 surface, and, more generally, any compact hyperkähler manifold is never Moishezon.

Theorem 4.11: Let $M$ be a compact hyperkähler manifold, and $\operatorname{Tw}(M)$ its twistor space. Then $\operatorname{deg}_{t r} \operatorname{Mer}(\operatorname{Tw}(M))=1$.

Proof: Let $Z \subset \operatorname{Tw}(M)$ be any divisor, and $R \subset \mathbb{C} P^{1}$ a countable subset constructed in Theorem 2.6. For any induced complex structure $L \notin R$, all complex subvarieties of $(M, L)$ are even-dimensional. Therefore, $Z$ intersects the twistor fiber $(M, L)=\pi^{-1}(L) \subset \operatorname{Tw}(M)$ non-transversally, or not at all. By Thom's transversality theorem, the intersection $Z$ with all fibers of $\pi$ except a finite number is transversal. This means that $Z$ can intersect only finitely many of the fibers of $\pi$. However, all these fibers are irreducible divisors. Therefore, $D$ is a union of several fibers of $\pi$. Since a meromorphic function is uniquely determined by its pole or zero divisor, all meromorphic functions on $\operatorname{Tw}(M)$ are pull-backs of meromorphic functions on $\mathbb{C} P^{1}$.

Remark 4.12: When $M$ is hypercomplex, no effective bounds on the transcendence degree $\operatorname{deg}_{t r} \operatorname{Mer}(\operatorname{Tw}(M))$ are known. We conjecture (based on empirical evidence) that the twistor space of a compact hypercomplex manifold is not Moishezon, but this conjecture seems to be difficult. 


\subsection{Twistor spaces and hyperkähler reduction}

We recall the definition of hyperkähler reduction, following [HKLR] and [Nak]. This material is fairly standard.

We denote the Lie derivative along a vector field as $\operatorname{Lie}_{x}: \Lambda^{i} M \longrightarrow \Lambda^{i} M$, and contraction with a vector field by $i_{x}: \Lambda^{i} M \longrightarrow \Lambda^{i-1} M$. Recall the Cartan's formula:

$$
d \circ i_{x}+i_{x} \circ d=\operatorname{Lie}_{x}
$$

Let $(M, \omega)$ be a symplectic manifold, $G$ a Lie group acting on $M$ by symplectomorphisms, and $\mathfrak{g}$ its Lie algebra. For any $g \in \mathfrak{g}$, denote by $\rho_{g}$ the corresponding vector field. Cartan's formula gives $\operatorname{Lie}_{\rho_{g}} \omega=0$, hence $d\left(i_{\rho_{g}}(\omega)\right)=0$. We obtain that $i_{\rho_{g}}(\omega)$ is closed, for any $g \in \mathfrak{g}$.

Definition 4.13: A Hamiltonian of $g \in \mathfrak{g}$ is a function $h$ on $M$ such that $d h=i_{\rho_{g}}(\omega)$.

Definition 4.14: $(M, \omega)$ be a symplectic manifold, $G$ a Lie group acting on $M$ by symplectomorphisms. A moment map $\mu$ of this action is a linear map $\mathfrak{g} \longrightarrow C^{\infty} M$ associating to each $g \in \mathfrak{g}$ its Hamiltonian.

Remark 4.15: It is more convenient to consider $\mu$ as an element of $\mathfrak{g}^{*} \otimes_{\mathbb{R}} C^{\infty} M$, or, as it is usually done, a function on $M$ with values in $\mathfrak{g}^{*}$.

Remark 4.16: Note that the moment map always exists, if $M$ is simply connected.

Definition 4.17: A moment map $M \longrightarrow \mathfrak{g}^{*}$ is called equivariant if it is equivariant with respect to the coadjoint action of $G$ on $\mathfrak{g}^{*}$. 
Remark 4.18: $M \stackrel{\mu}{\longrightarrow} \mathfrak{g}^{*}$ is a moment map if and only if for all $g \in \mathfrak{g},\langle d \mu, g\rangle=$ $i_{\rho_{g}}(\omega)$. Therefore, a moment map is defined up to a constant $\mathfrak{g}^{*}$-valued function. An equivariant moment map is is defined up to a constant $\mathfrak{g}^{*}$-valued function which is $G$-invariant.

Definition 4.19: A $G$-invariant $c \in \mathfrak{g}^{*}$ is called central.

Claim 4.20: An equivariant moment map exists whenever $H^{1}\left(G, \mathfrak{g}^{*}\right)=0$. In particular, if $G$ is reductive and $M$ is simply connected, an equivariant moment map is always possible to define.

Definition 4.21: Let $(M, \omega)$ be a symplectic manifold, $G$ a compact Lie group acting on $M$ by symplectomorphisms, $M \stackrel{\mu}{\longrightarrow} \mathfrak{g}^{*}$ an equivariant moment map, and $c \in \mathfrak{g}^{*}$ a central element. The quotient $\mu^{-1}(c) / G$ is called the symplectic reduction of $M$, denoted by $M / / G$.

Claim 4.22: The symplectic quotient $M / / G$ is a symplectic manifold of dimension $\operatorname{dim} M-2 \operatorname{dim} G$.

Theorem 4.23: Let $(M, I, \omega)$ be a Kähler manifold, $G_{\mathbb{C}}$ a complex reductive Lie group acting on $M$ by holomorphic automorphisms, and $G$ is a compact form of $G_{\mathbb{C}}$ acting isometrically. Then $M / / G$ is a Kähler orbifold.

Remark 4.24: In such a situation, $M / / G$ is called the Kähler quotient, or GIT quotient.

Remark 4.25: The points of $M / / G$ are in bijective correspondence with the orbits of $G_{\mathbb{C}}$ which intersect $\mu^{-1}(c)$. Such orbits are called polystable, and the 
intersection of a $G_{\mathbb{C}^{-}}$orbit with $\mu^{-1}(c)$ is a $G$-orbit.

Definition 4.26: Let $G$ be a compact Lie group, $\rho$ its action on a hyperkähler manifold $M$ by hyperkähler isometries, and $\mathfrak{g}^{*}$ a dual space to its Lie algebra. A hyperkähler moment map is a $G$-equivariant smooth map $\mu: M \rightarrow \mathfrak{g}^{*} \otimes \mathbb{R}^{3}$ such that $\left\langle\mu_{i}(v), g\right\rangle=\omega_{i}(v, d \rho(g))$, for every $v \in T M, g \in \mathfrak{g}$ and $i=1,2,3$, where $\omega_{i}$ are three Kähler forms associated with the hyperkähler structure.

Definition 4.27: Let $\xi_{1}, \xi_{2}, \xi_{3}$ be three $G$-invariant vectors in $\mathfrak{g}^{*}$. The quotient manifold $M / / / G:=\mu^{-1}\left(\xi_{1}, \xi_{2}, \xi_{3}\right) / G$ is called the hyperkähler quotient of $M$.

Theorem 4.28: ([HKLR]) The quotient $M / / / G$ is hyperkaehler.

Proof: We sketch the proof of Hitchin-Karlhede-Lindström-Roček theorem, because we make use of it further on.

Let $\Omega:=\omega_{J}+\sqrt{-1} \omega_{K}$. This is a holomorphic symplectic $(2,0)$-form on $(M, I)$. Let $\mu_{J}, \mu_{K}$ be the moment map associated with $\omega_{J}, \omega_{K}$, and $\mu_{\mathbb{C}}:=\mu_{J}+\sqrt{-1} \mu_{K}$. Then $\left\langle d \mu_{\mathbb{C}}, g\right\rangle=i_{\rho_{g}}(\Omega)$ Therefore, $d \mu_{\mathbb{C}} \in \Lambda^{1,0}(M, I) \otimes \mathfrak{g}^{*}$. This implies that the map $\mu_{\mathbb{C}}$ is holomorphic. It is called a holomorphic moment map.

By definition, $M / / / G=\mu_{\mathbb{C}}^{-1}(c) / / G$, where $c \in \mathfrak{g}^{*} \otimes_{\mathbb{R}} \mathbb{C}$ is a central element. This is a Kähler manifold, because it is a Kähler quotient of a Kähler manifold.

We obtain 3 complex structures $I, J, K$ on the hyperkähler quotient $M / / / G$. They are compatible in the usual way, as seen from a simple local computation.

Theorem 4.29: Let $V$ be a quaterionic Hermitian vector space, and $G \subset \operatorname{Sp}(V)$ a compact Lie group acting on $V$ by quaternionic isometries. Denote by $M$ the hyperkähler reduction of $V$. Then $\operatorname{Tw}(M)$ is a Moishezon twistor space, in the sense of Definition 4.9. 
Proof: The holomorphic symplectic form on $(V, I)$ depends on $I$ holomorphically, giving a section

$$
\Omega_{t w} \in \Omega_{\pi}^{2}(\mathrm{Tw}(V)) \otimes_{\mathcal{O}_{\mathrm{Tw}(V)}} \pi^{*} \mathcal{O}(2) .
$$

Here, $\pi: \operatorname{Tw}(V) \longrightarrow \mathbb{C} P^{1}$ is the twistor projection, and $\Omega_{\pi}^{2}(\operatorname{Tw}(V))$ the sheaf of fiberwise holomorphic 2 -forms. Consider the fiberwise holomorphic moment map given by this form, $\mu_{t w}: \operatorname{Tw}(V) \longrightarrow \operatorname{Tot}\left(\mathfrak{g}^{*} \otimes_{\mathbb{C}} \mathcal{O}(2)\right)$. Replacing the moment map by its translate, we can always assume that $M / / / G=\mu_{\mathbb{C}}^{-1}(0) / / G$ (that is, we assume that the central vector $c$ used to define $M / / / G$ vanishes). Then $\operatorname{Tw}(M)$

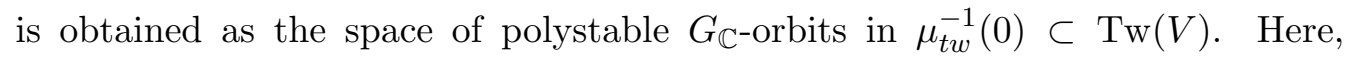
"polystability" of an orbit is understood as the non-emptiness of the intersection of this orbit with $\mu^{-1}(0)$; the set of such orbits is open.

The space $\operatorname{Tw}(V)=\mathbb{C} P^{2 n+1} \backslash \mathbb{C} P^{2 n-1}$ is quasiprojective. Averaging the ring

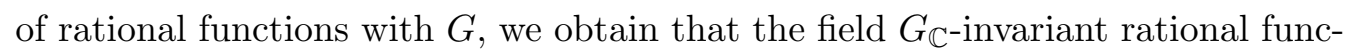
tions on $\operatorname{Tw}(V)$ has dimension $\operatorname{dim} \mu_{t w}^{-1}(0)-\operatorname{dim} G_{\mathbb{C}}=\operatorname{dim} \operatorname{Tw}(M)$, and hence $\operatorname{Tw}(M)$ is Moishezon.

Corollary 4.30: Let $U$ be an open subset of a compact, simply connected hyperkähler manifold, and $U^{\prime}$ an open subset of a hyperkähler manifold obtained as $V / / / G$, where $V$ is flat and $G$ reductive. Then $U$ is not isomorphic to $U^{\prime}$ as hyperkähler manifold.

Proof: $\operatorname{dim}_{t r} \operatorname{Tw}\left(U^{\prime}\right)=\operatorname{dim} \operatorname{Tw}\left(U^{\prime}\right)$ by Theorem 4.29, and $\operatorname{dim}_{t r} \operatorname{Tw}(U)=1$ by Theorem 4.11 .

A twistor space of a manifold obtained by hyperkähler reduction is Moishezon, and from the above argument it is easy to see that it is Zariski open in a compact Moishezon variety. However, it is (almost) never quasiprojective.

Theorem 4.31: Let $M$ be a hyperkähler manifold such that its twistor space $\operatorname{Tw}(M)$ can be embedded to a projective manifold. Then, for each induced com- 
plex structure $L$, the complex manifold $(M, L)$ has no compact subvarieties of positive dimension.

Proof: Consider the anticomplex involution $\iota$ on $\operatorname{Tw}(M)$ mapping $(m, L)$ to $(m,-L)$. Suppose that $(M, L) \subset \operatorname{Tw}(M)$ has a compact subvariety. Since the $(M, L)$ is quasiprojective, this would imply that $(M, L)$ contains a compact curve $S$. The curve $\iota(S)$ is also holomorphic in $(M,-L)$.

Consider a Kähler form $\omega$ on $\operatorname{Tw}(M)$. Since $\iota$ is antiholomorphic, $-\iota(\omega)$ is a closed, positive $(1,1)$-form. Replacing $\omega$ by $\omega-\iota(\omega)$, we may assume that $\omega$ satisfies $\iota(\omega)=-\omega$.

Now, since the cohomology classes of $S$ and $\iota(S)$ are equal, we have

$$
\int_{S} \omega=\int_{\iota(S)} \omega=-\int_{\iota(S)} \iota(\omega)
$$

On the other hand, $\int_{S} \omega=\int_{\iota(S)} \iota(\omega)$ by functorial properties of integral. This implies $\int_{S} \omega=0$, giving a contradiction.

Remark 4.32: As shown in [V3], a general fiber of the map $\operatorname{Tw}(M) \longrightarrow \mathbb{C} P^{1}$ has only even-dimensional complex subvarieties.

\section{Appendix: Formal geometry and holography prin- ciple (by Dmitry Kaledin)}

In this Appendix, we will try to explain the constructions of the paper in a slightly more general context of $\mathcal{D}$-modules and "formal geometry" of Gelfand and Kazhdan [GK]. To save space, we only sketch the proofs, and we work in the algebraic setting (generalization to complex-analytic varieties is immediate, exactly the same arguments work).

Assume given a smooth algebraic variety $X$ over a field $k$ of characteristic 0 . We will work with coherent $\mathcal{D}$-modules over $X$, that is, with sheaves of left modules over the algebra $\mathcal{D}_{X}$ of differential operators on $X$ which are finitely 
generated over $\mathcal{D}$. Any coherent $\mathcal{D}$-module $\mathcal{E}$ is also a quasicoherent sheaf of $\mathcal{O}_{X}$-modules; recall that if $\mathcal{E}$ is coherent over $\mathcal{O}_{X}$, then it comes from a vector bundle on $X$ equipped with a flat connection. In particular, the structure sheaf $\mathcal{O}_{X}$ is a $\mathcal{D}$-module; it corresponds to the trivial line bundle. For any coherent sheaf $\mathcal{E}$ on $X$, we can consider the induced $\mathcal{D}$-module $\mathcal{D}_{X} \otimes_{\mathcal{O}_{X}} \mathcal{E}$; this is coherent over $\mathcal{D}_{X}$ but not over $\mathcal{O}_{X}$. Coherent $\mathcal{D}$-modules form an abelian category, and we can consider its derived category.

Assume given an open subvariety $U \subset X$ with the embedding map $j: U \rightarrow X$, and let $Z=X \backslash U \subset X$ be the complement $U$. Then the complex of quascoherent sheaves $R^{*} j_{*} \mathcal{O}_{U}$ on $X$ has a natural structure of a complex of $\mathcal{D}$-modules, and we have an exact triangle

$$
\delta_{Z} \longrightarrow \mathcal{O}_{X} \longrightarrow R^{\bullet} j_{*} \mathcal{O}_{X} \longrightarrow
$$

of complexes of $\mathcal{D}$-modules on $X$, where $\delta_{Z}$ is supported at $Z$. We will need the following standard result.

Lemma 5.1: In the notation above, for any coherent sheaf $\mathcal{E}$ on $X$, we have a natural identification

$$
\operatorname{Hom}_{\mathcal{O}_{X}}\left(\delta_{Z}, \mathcal{E}\right) \cong \Gamma\left(X, \mathcal{E} \otimes \widehat{\mathcal{O}}_{X, Z}\right),
$$

where $\widehat{\mathcal{O}}_{X, Z}$ is the formal completion of structure sheaf $\mathcal{O}_{X}$ at the closed subscheme $Z \subset X$.

Sketch of a proof. Since $\delta_{Z}$ is supported at $Z$, the natural map

$$
\operatorname{Hom}_{\mathcal{O}_{X}}\left(\delta_{Z}, \mathcal{E}\right) \rightarrow \operatorname{Hom}_{\mathcal{O}_{X}}\left(\delta_{Z}, \mathcal{E} \otimes \widehat{\mathcal{O}}_{X, Z}\right)
$$

is an isomorphism. On the other hand, we obviously have

$$
\operatorname{Hom}_{\mathcal{O}_{X}}\left(R^{\bullet} j_{*} \mathcal{O}_{U}, E \otimes \widehat{\mathcal{O}}_{X, Z}\right)=0,
$$

and the claim then immediately follows from the long exact sequence associated to the exact triangle (5.1).

For any map $f: X \rightarrow Y$ of smooth algebraic varieties, the pullback functor $f^{*}$ extends to a functor between $\mathcal{D}$-modules, and its derived functor extends to 
a functor between the derived categories of $\mathcal{D}$-modules; we will denote this last functor by $f$ ? We have

$$
f^{?}=f^{*}[\operatorname{dim} X-\operatorname{dim} Y]
$$

where $f^{*}$ is the standard pullback functor for $\mathcal{D}$-modules, and [-] stands for cohomological shift. If $f$ is a smooth map, or a closed embedding, or a composition of the two, then $f$ ? has a left-adjoint functor $f$ ? given by

$$
f_{?}=f_{!}[\operatorname{dim} Y-\operatorname{dim} X],
$$

where $f$ i is the standard functor of direct image with compact supports. Then Lemma 5.1 has the following corollary.

Lemma 5.2: Assume given a map $f: Y \rightarrow X$ of smooth algebraic varieties, and assume that $f$ factors as

$$
Y \stackrel{\pi}{\longrightarrow} Z \stackrel{\iota}{\longrightarrow} X
$$

where $Z$ is smooth, $\iota$ is a closed embedding, and $\pi$ is a smooth map with contractible fibers. Then for any vector bundle $\mathcal{E}$ on $X$, we have a natural identification

$$
\operatorname{Hom}_{\mathcal{D}_{Y}}\left(f^{?}\left(\mathcal{D}_{X} \otimes_{\mathcal{O}_{X}} \mathcal{E}^{*}\right), \mathcal{O}_{Y}\right) \cong \Gamma\left(X, \mathcal{E} \otimes \widehat{\mathcal{O}}_{X, Z}\right),
$$

where $\mathcal{E}^{*}$ is the dual vector bundle, and $\mathcal{D}_{X} \otimes_{\mathcal{O}_{X}} \mathcal{E}^{*}$ is the corresponding induced $\mathcal{D}$-module.

Proof. For any $\mathcal{D}$-module $\mathcal{F}$ and $\mathcal{O}_{X}$-module $\mathcal{E}$, we have

$$
\operatorname{Hom}_{\mathcal{D}_{X}}\left(\mathcal{D}_{X} \otimes_{\mathcal{O}_{X}} \mathcal{E}, \mathcal{F}\right) \cong \operatorname{Hom}_{\mathcal{O}_{X}}(\mathcal{E}, F),
$$

so that Lemma 5.1 provides an identification

$$
\operatorname{Hom}_{\mathcal{D}_{X}}\left(\mathcal{D}_{X} \otimes_{\mathcal{O}_{X}} \mathcal{E}^{*} \otimes_{\mathcal{O}_{X}} \delta_{Z}, \mathcal{O}_{X}\right) \cong \Gamma\left(X, \mathcal{E} \otimes \widehat{\mathcal{O}}_{X, Z}\right),
$$

and by adjunction, it suffices to construct an isomorphism

$$
f_{?} f^{?}\left(\mathcal{D}_{X} \otimes_{\mathcal{O}_{X}} \mathcal{E}^{*}\right) \cong \mathcal{D}_{X} \otimes_{\mathcal{O}_{X}} \mathcal{E}^{*} \otimes_{\mathcal{O}_{X}} \delta_{Z}
$$


But under our assumptions on $f$, we have $f_{?} \mathcal{O}_{X} \cong \delta_{Z}$, and we are done by the projection formula.

We note that the left-hand side of (5.2) admits a slightly different interpretation. Recall that for any coherent sheaf $\mathcal{E}$ on $X$, the jet bundle $J^{\infty} \mathcal{E}$ of $\mathcal{E}$ is a (pro)coherent sheaf on $X$ given by

$$
J^{\infty} \mathcal{E}=\pi_{2 *} \pi_{1}^{*} \mathcal{E}
$$

where $\pi_{1}, \pi_{2}: \widehat{X} \rightarrow X$ are the two natural projections of the completion $\widehat{X}$ of the product $X \times X$ near the diagonal $X \subset X \times X$. In terms of $\mathcal{D}$-modules, we have

$$
J^{\infty} \mathcal{E} \cong \mathcal{H}_{\mathrm{om}_{\mathcal{O}_{X}}}\left(\mathcal{D}_{X}, \mathcal{E}\right)
$$

The jet bundle $J^{\infty} \mathcal{E}$ carries a canonical flat connection, and in the assumptions of Lemma 5.2, we have

$$
\begin{aligned}
\operatorname{Hom}_{\mathcal{D}_{Y}}\left(f^{?}\left(\mathcal{D}_{X} \otimes_{\mathcal{O}_{X}} \mathcal{E}^{*}\right), \mathcal{O}_{Y}\right) & \cong \operatorname{Hom}_{\mathcal{D}_{Y}}\left(\mathcal{O}_{Y}, \mathcal{H} \operatorname{lom}_{\mathcal{O}_{Y}}\left(f^{?}\left(\mathcal{D}_{X} \otimes_{\mathcal{O}_{X}} \mathcal{E}^{*}\right), \mathcal{O}_{Y}\right)\right) \\
& \cong \operatorname{Hom}_{\mathcal{D}_{Y}}\left(\mathcal{O}_{Y}, f^{?}\left(\mathcal{H o m}_{\mathcal{O}_{X}}\left(\mathcal{D}_{X} \otimes_{\mathcal{O}_{X}} \mathcal{E}^{*}, \mathcal{O}_{X}\right)\right)\right) \\
& \cong \Gamma^{\nabla}\left(Y, f^{*} J^{\infty} \mathcal{E}\right),
\end{aligned}
$$

where $\Gamma^{\nabla}(-)$ stands for the space of flat global sections. Then (5.2) reads as

$$
\Gamma^{\nabla}\left(Y, f^{*} J^{\infty} \mathcal{E}\right) \cong \Gamma\left(X, \mathcal{E} \otimes \widehat{\mathcal{O}}_{X, Z}\right)
$$

Remark 5.3: The assumptions of Lemma 5.2 are in fact too strong. Firstly, it is clearly enough to require that the fibers of $\pi$ are non-empty and connected, so that their top degree cohomology with compact supports is one-dimensional - and under the assumptions as stated, we not only obtain an isomorphism of Hom's but also of the RHom's, so that (5.3) extends to an isomorphism

$$
H_{D R}^{\cdot}\left(Y, f^{*} J^{\infty} \mathcal{E}\right) \cong H^{\bullet}\left(X, \mathcal{E} \otimes \widehat{\mathcal{O}}_{X, Z}\right)
$$

of cohomology groups. Secondly, one probably does not have to require that $\pi$ and $Z$ are smooth - some assumptions are needed, but they can considerably relaxed. However, since even the stronger assumptions work for us, we did not pursue this. 
Assume now that we are given smooth proper algebraic varieties $Y, X$, and a family of closed embedding from $Y$ to $X$ parametrized by a smooth algebraic variety $T$ - that is, we have a map

$$
f: Y \times T \rightarrow X
$$

such that for any $t \in T$, the corresponding map $f_{t}: Y=Y \times t \rightarrow X$ is a closed embedding. Moreover, assume given a coherent sheaf $\mathcal{E}$ on $X$, denote by $\rho: Y \times T \rightarrow T$ the projection, and denote

$$
\Phi_{f} \mathcal{E}=\rho_{*}^{\nabla} f^{*} J^{\infty} \mathcal{E},
$$

where $f^{*} J^{\infty} \mathcal{E}$ is the pullback of the jet bundle $J^{\infty} \mathcal{E}$ equipped with its natural flat connection $\nabla$, and $\rho_{*}^{\nabla}$ stands for sheaf of relative flat sections. Then $\Phi_{a} \mathcal{E}$ is a sheaf on $T$, the base of the family, and by (5.3), the fiber $\left(\Phi_{f} \mathcal{E}\right)_{t}$ at a point $t \in T$ is given by

$$
\left(\Phi_{f} \mathcal{E}\right)_{t}=\Gamma^{\nabla}\left(Y, f_{t}^{*} J^{\infty} \mathcal{E}\right) \cong \Gamma\left(X, \mathcal{E} \otimes \widehat{\mathcal{O}}_{X, Y_{t}}\right)
$$

where $Y_{t} \subset X$ is the image of the closed embedding $f_{t}: Y \rightarrow X$. On the other hand, $\Phi_{f} \mathcal{E}$ carries a natural flat connection, and if we assume that $f$ is smooth with contractible fibers, we have

$$
\Gamma^{\nabla}\left(T, \Phi_{f} \mathcal{E}\right)=\Gamma^{\nabla}\left(T \times Y, f^{*} J^{\infty} \mathcal{E}\right) \cong \Gamma(X, \mathcal{E}),
$$

again by (5.3).

We now note that this is exactly the situation that we have in the paper. Namely, we take $X$ to be the twistor space of a hyperkähler manifold $M$, we take $T=M_{\mathbb{C}}$ to be the complexification of the real-analytic manifold underlying $M$, we take $Y=\mathbb{C} P^{1}$, and we let

$$
f: T \times Y \rightarrow X
$$

be the standard family of twistor lines (real points in this family correspond to horizontal sections of the twistor fibration $X \rightarrow \mathbb{C} P^{1}$ parametrized by points of $M)$. Then since the normal bundle to any line in our family is a sum of several copies of $\mathcal{O}(1)$, the family is unobstructed, and moreover, it remains unobstructed 
even if we fix a point at a twistor line, so that the map $f$ is smooth. Its fibers are small polydiscs, thus contractible, and all the assumptions of Lemma 5.2 are therefore satisfied.

\section{References}

[BBI] Bădescu, Lucian; Beltrametti, Mauro C.; Ionescu, Paltin, Almost-lines and quasilines on projective manifolds, Complex analysis and algebraic geometry, 1-27, de Gruyter, Berlin, 2000.

[AHS] Atiyah, M. F., Hitchin, N. J., and Singer, I. M. Self-duality in four-dimensional Riemannian geometry, Proc. Roy. Soc. London Ser. A 362 (1978), 425-461.

[Bes] Besse, A., Einstein Manifolds, Springer-Verlag, New York (1987).

[C] F. Campana, On twistor spaces of the class $\mathcal{C}$, J. Differential Geom. 33 (1991) 541-549.

[D] Demailly, Jean-Pierre, Pseudoconvex-concave duality and regularization of currents, Several complex variables (Berkeley, CA, 1995-1996), 233-271, Math. Sci. Res. Inst. Publ., 37, Cambridge Univ. Press, Cambridge, 1999.

[GK] I.M. Gelfand and D.A. Kazhdan, Some problems of differential geometry and the calculation of cohomologies of Lie algebras of vector fields, Soviet Math. Dokl. 12 (1971), 1367-1370.

[Har] Hartshorne, Robin, Cohomological dimension of algebraic varieties, Ann. of Math. (2) 881968 403-450.

[HKLR] N. J. Hitchin, A. Karlhede, U. Lindström, M. Roček, Hyperkähler metrics and supersymmetry, Comm. Math. Phys. 108, (1987) 535-589.

[Ho] Nobuhiro Honda Moishezon twistor spaces on $4 \mathbb{C} P^{2}$, arXiv:1112.3109, 51 pages.

[K] D. Kaledin, Integrability of the twistor space for a hypercomplex manifold, Sel. math., New ser. 4 (1998) 271-278.

[KV] Kaledin, D., Verbitsky, M., Non-Hermitian Yang-Mills connections, Selecta Math. (N.S.) 4 (1998), no. 2, 279-320.

[KST] Stefan Kebekus, Luis Sola Conde, Matei Toma, Rationally connected foliations after Bogomolov and McQuillan, J. Algebraic Geom. 16 (2007), no. 1, 65-81. 
[Ko] Kollár, J., Rational curves on algebraic varieties, Springer, 1996, viii+320 pp..

[Nak] H. Nakajima, Lectures on Hilbert schemes of points on surfaces, Providence: American Mathematical Society, 1999.

[P] Y. S. Poon, On the algebraic structure of twistor spaces, J. Diff. Geom. 36 (1992), 451-491.

[SV] Andrey Soldatenkov, Misha Verbitsky, Subvarieties of hypercomplex manifolds with holonomy in $S L(n, \mathbb{H})$, Journal of Geometry and Physics, Volume 62, Issue 11 (2012), Pages 2234-2240, arXiv:1202.0222

[V1] Verbitsky M., Tri-analytic subvarieties of hyper-Kaehler manifolds, also known as Hyperkähler embeddings and holomorphic symplectic geometry II, GAFA 5 no. 1 (1995), 92-104, alg-geom/9403006.

[V2] Verbitsky, M., Hypercomplex Varieties, alg-geom/9703016, Comm. Anal. Geom. 7 (1999), no. 2, 355-396.

[V3] Verbitsky, M., Subvarieties in non-compact hyperkähler manifolds, Math. Res. Lett., vol. 11 (2004), no. 4, pp. 413-418.

[V4] Verbitsky, M., Rational curves and special metrics on twistor spaces, arXiv:1210.6725, 12 pages.

[Y] Yau, S. T., On the Ricci curvature of a compact Kähler manifold and the complex Monge-Ampère equation I. Comm. on Pure and Appl. Math. 31, 339-411 (1978).

Misha Verbitsky

Laboratory of Algebraic Geometry,

Faculty of Mathematics, National Research University HSE,

7 Vavilova Str. Moscow, Russia

E-mail: verbit@verbit.ru 